\title{
Effect of 8-Week Combined Training (Resistance and Proprioceptive Neuromuscular Facilitation) on Fatigue and Quality of Life in Multiple Sclerosis Patients
}

\author{
Attar Sayyah A.E.* MSc, Hoseini Kakhk S.A.R. ${ }^{1}$ PhD, Hamedinia M.R. ${ }^{1}$ PhD, Mehrjoo M. ${ }^{2}$ MSc
}

*Department of Exercise Physiology, Faculty of Physical Education \& Sports Science, Hakim Sabzevary University, Sabzevar, Iran

1Department of Exercise Physiology, Faculty of Physical Education \& Sports Science, Hakim Sabzevary University, Sabzevar, Iran

${ }^{2}$ General Department, Faculty of Physical Education \& Sports Science, Hakim Sabzevary University, Sabzevar, Iran

\begin{abstract}
Aims: Multiple sclerosis (MS) is a chronic disease of central nervous system that affects motor pathways and increase fatigue and decrease quality of life. The purpose of this research was to analyze the effect of combined (resistance and proprioceptive neuromuscular facilitation) training for 8 weeks on fatigue and quality of life in multiple sclerosis patients.

Materials \& Methods: This semi-experimental study was done in 2013. Samples were 37 women and men patients with multiple sclerosis which were divided to experimental $(n=19)$ and control $(n=18)$ groups. The experimental group participated in combined training program for 8 weeks. Fatigue and quality of life were evaluated before and after training protocol. Data were analyzed using independent and dependent T tests and MANOVA test with LSD post hoc.

Findings: 8 weeks of combined training decreased the fatigue and increased the quality of life and its subscales (physical health composite, mental health composite) significantly ( $\mathrm{p}=0.001)$.

Conclusion: Combined training causes decreasing of fatigue and increasing of quality of life in multiple sclerosis patients.
\end{abstract}

\section{Keywords}

Multiple Sclerosis [http://www.ncbi.nlm.nih.gov/mesh/68009103];

Quality of Life [http://www.ncbi.nlm.nih.gov/mesh/68011788];

Fatigue [http://www.ncbi.nlm.nih.gov/mesh/68005221]

\footnotetext{
* Corresponding Author

Tel: +985144012620

Fax: +985144012613

Address: Faculty of Physical Education \& Sports Science, Hakim Sabzevary University, Tohid Shahr, Sabzevar, Iran. Postal Code: 9617976487 
عصبى مركزى ايجاد مىشود. اين بيمارى يك اختلال التهابى

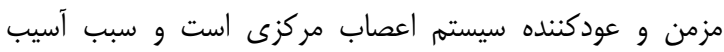

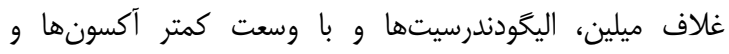

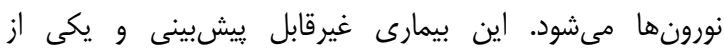

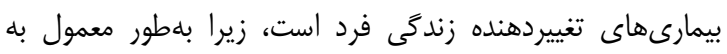

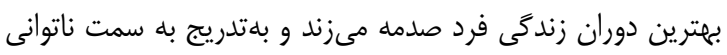
ييش مىرود و متاسفانه درمان قطعى ندارد[1].]

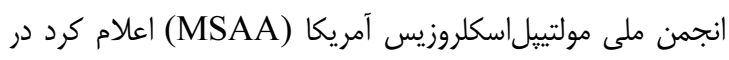

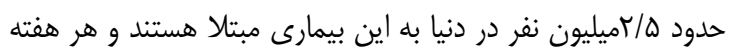

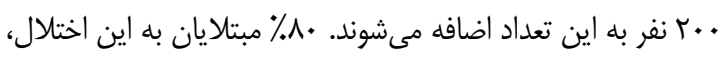

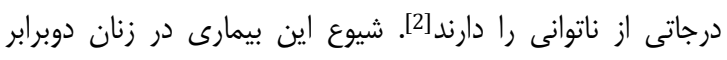

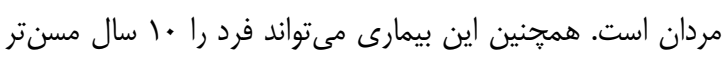

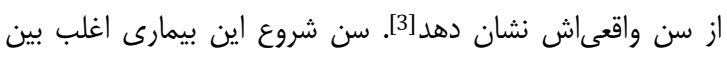

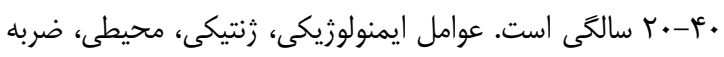

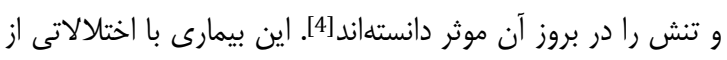

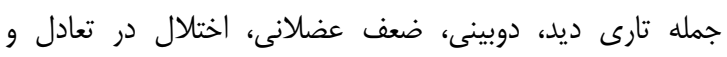

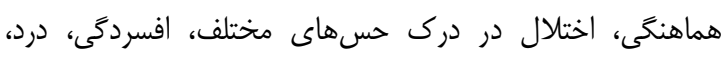

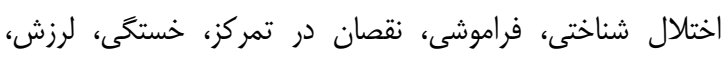

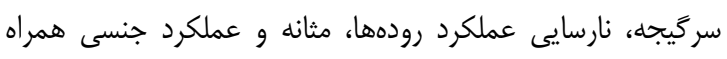

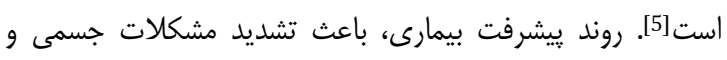

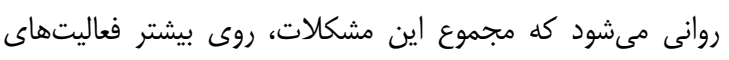

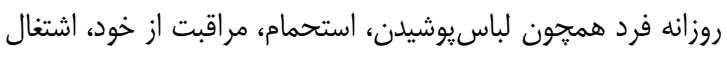

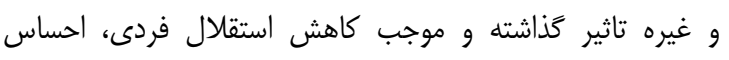

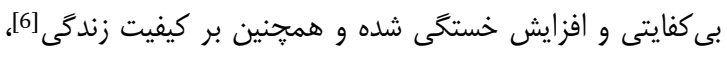

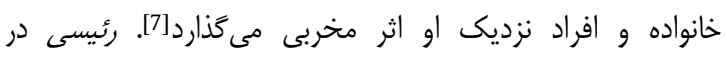

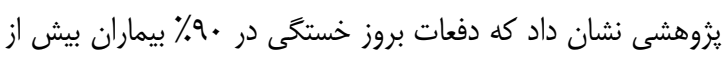

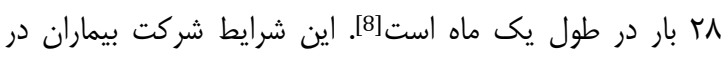

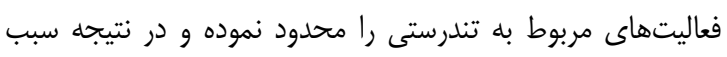

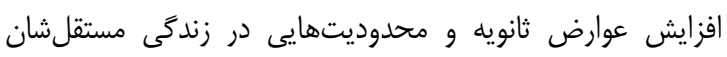

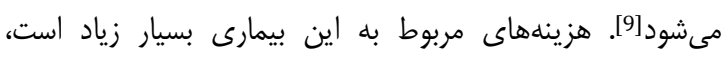

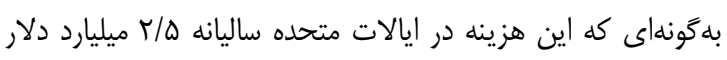

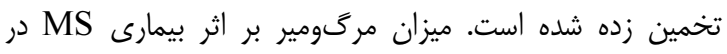

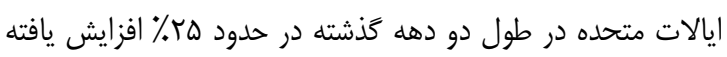

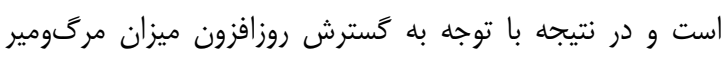

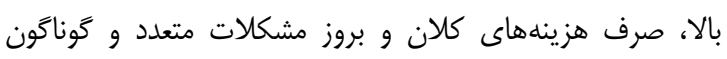

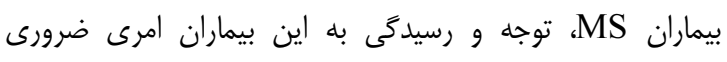

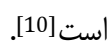

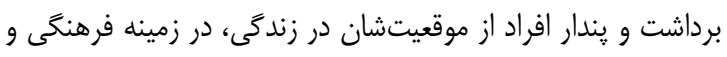

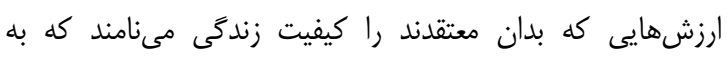

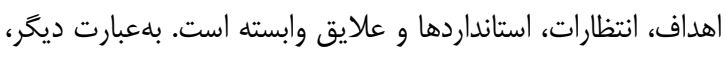

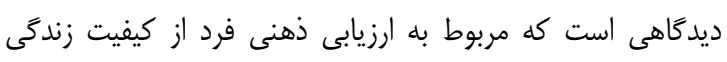

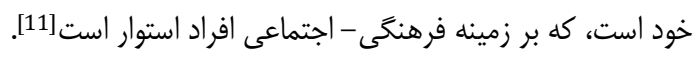

اثر هشت هفته تمرين تركيبى (مقاومتى و تسميل عصبى - عضلانى كَيرندههاى عمقى) بر ترئر

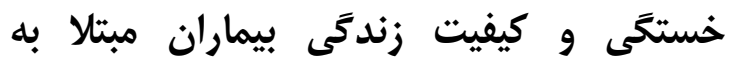
مولتيِل اسكلروزيس

MSc "اقابراهيم عطار سياح كروه فيزيولوزى ورزشى، دانشكده تربيت بدنى، دانشخاه حكيم سبزوارى، سبزوار، ايران

سيدعليرضا حسينى كاخك PhD كروه فيزيولوزى ورزشى، دانشكده تربيت بدنى، دانشكاه حكيم سبزوارى، سبزوار، ايران

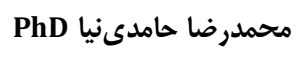
كَروه فيزيولوزى ورزشى، دانشكده تربيت بدنى، دانشخاه حكيم سبزوارى، سبزوار، ايران

مريم مهرجو MSc

كروه عمومى، دانشكده تربيت بدنى، دانشخاه حكيم سبزوارى، سبزوار، ايران جكيده اهداف: مولتييلاسكلروزيس بيمارى مزمن سيستم اعصاب مركزى است

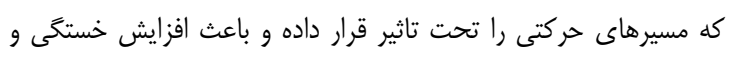

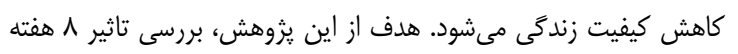

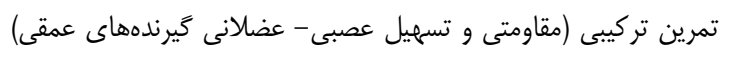

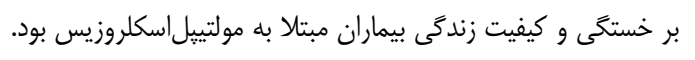

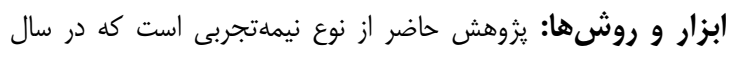

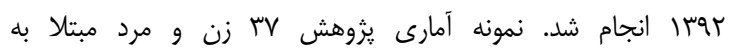

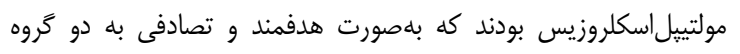

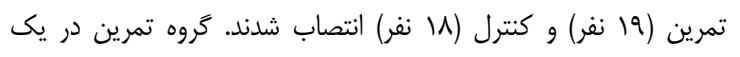

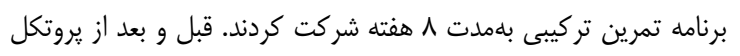

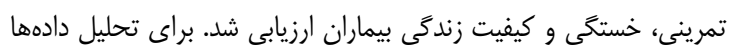

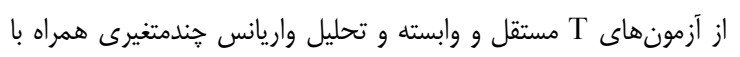

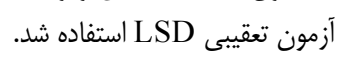

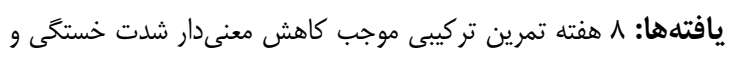

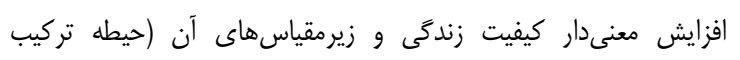

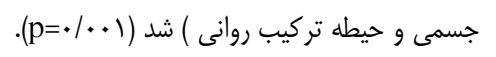

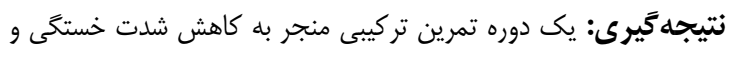

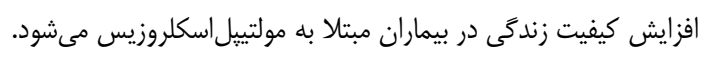

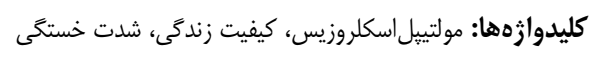

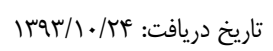
تاريخ پِيرش: "نويسنده مسئول: ebi_attar2000@yahoo.com

مقدمه

مولتييلاسكلروزيس (MS) شايعترين بيمارى ناتوان كننده افراد

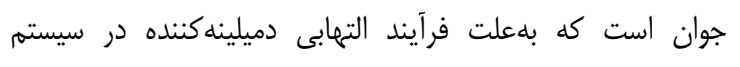

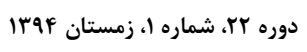
فصلنامه افق دانش 
Fه اثر هشت هفته تمرين تركيبى بر خستكى و كيفيت زندكى بيماران مبتلا به مولتييل اسكلروزيس

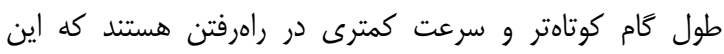

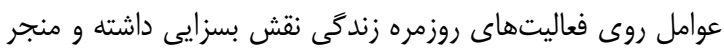

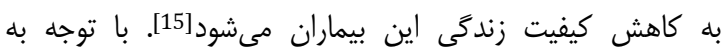

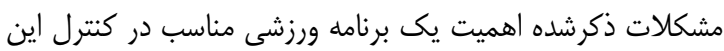

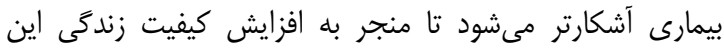

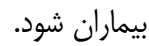

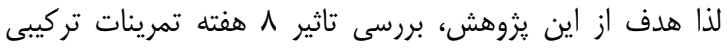

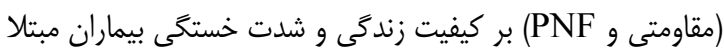

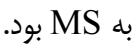

\section{ابزار و روشها}

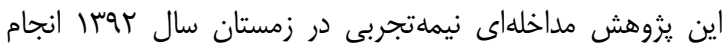

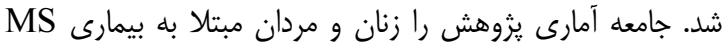

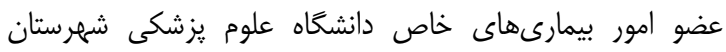

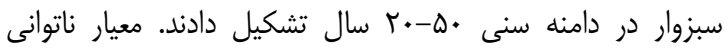

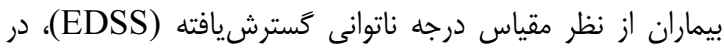

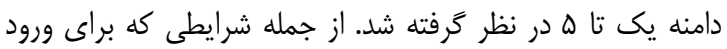

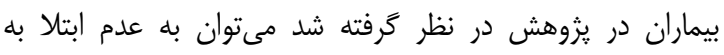

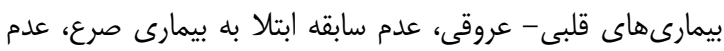

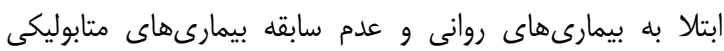

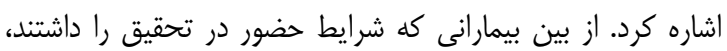

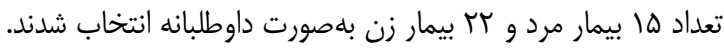

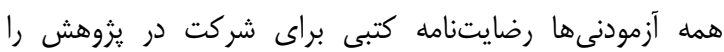

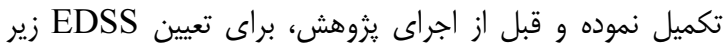

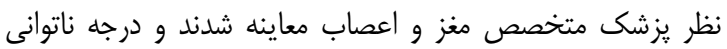

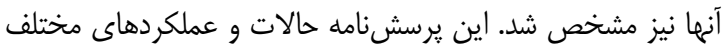

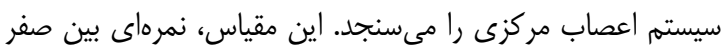

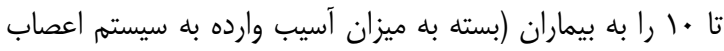

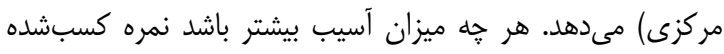

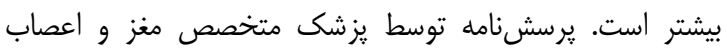
كامل شد. روايى آزمون ناتوانى جسمانى توسعهيافته كورتنز در ايران هنجار شده است. لازم به ذكر است كاهش

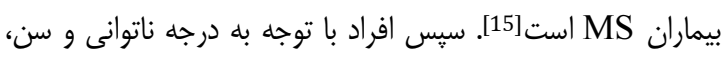

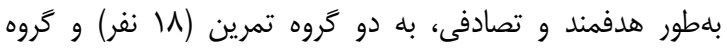
كنترل (19 نفر) تقسيم شدند.

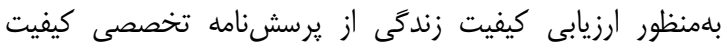

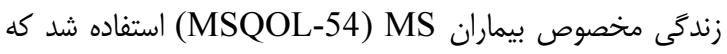

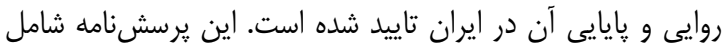

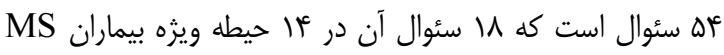
(سلامت جسمى، محدوديت ايفاى نقش مرتبط با وضعيت جسمى،

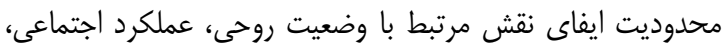

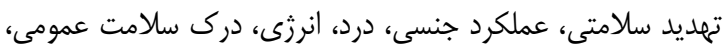

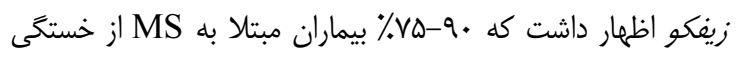

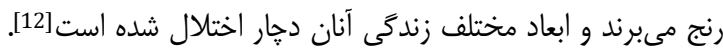

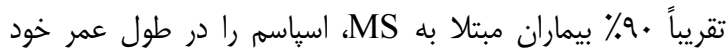

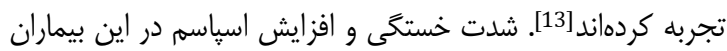

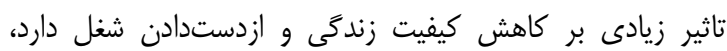

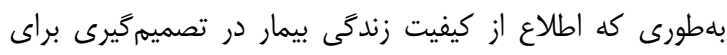

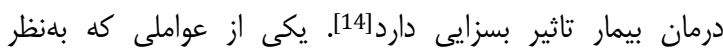

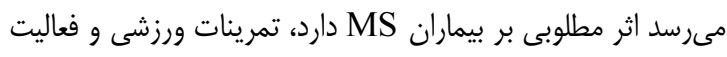

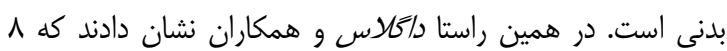

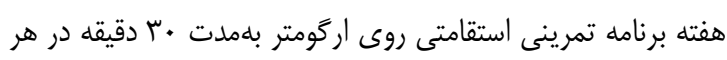

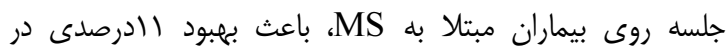

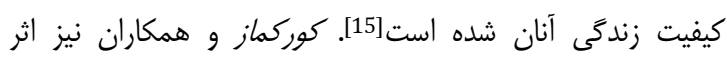

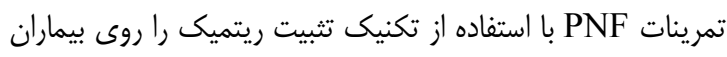

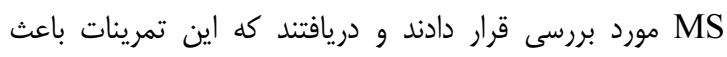

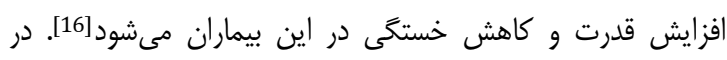

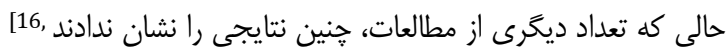

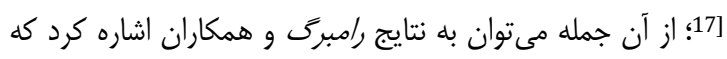

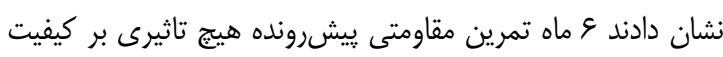

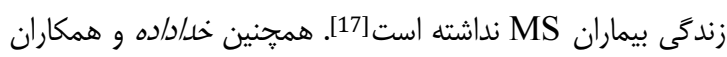

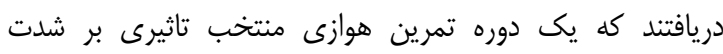

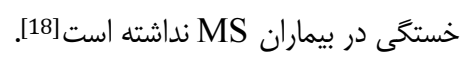

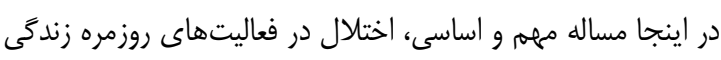

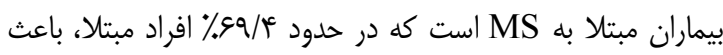

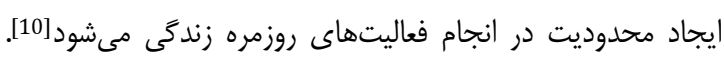

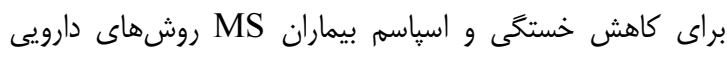

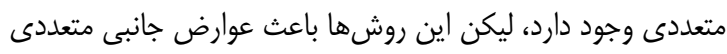

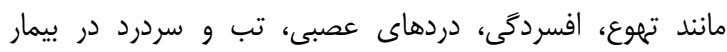

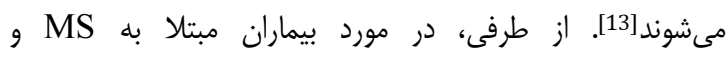

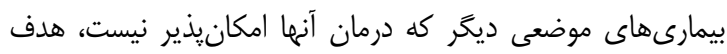

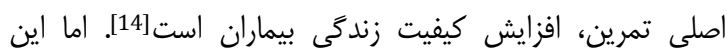

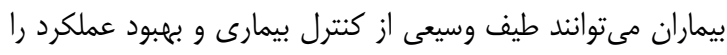

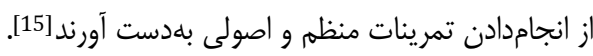

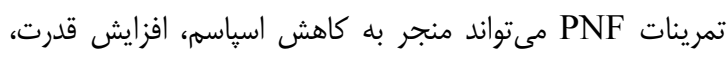

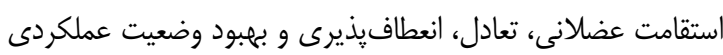

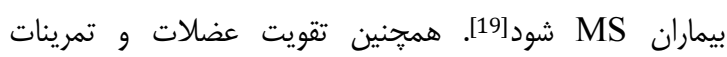

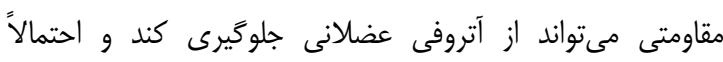

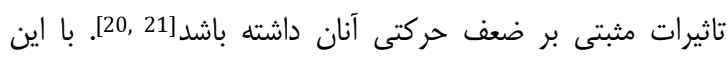

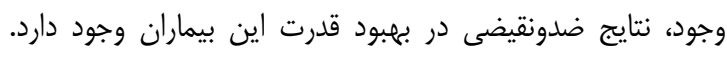

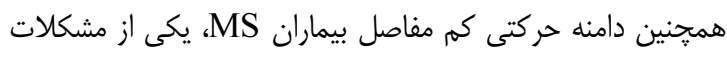

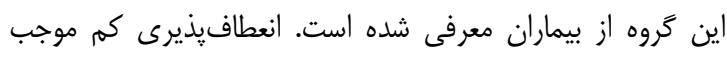

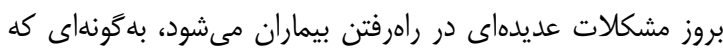

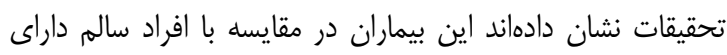




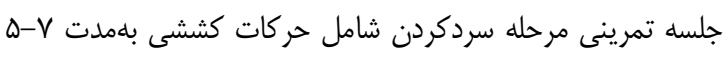

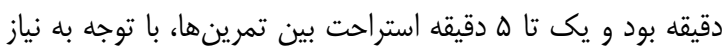

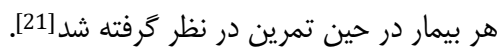

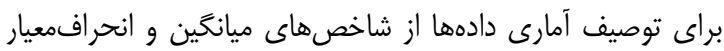

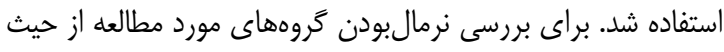

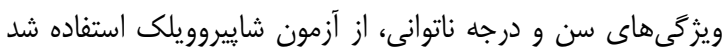

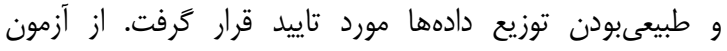

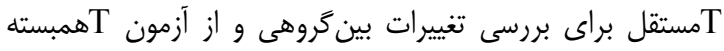
براى بررسى تغييرات درونگروهى در متغير شدت خستخى استفاده

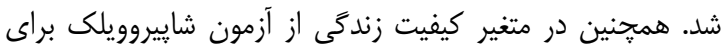

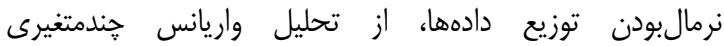

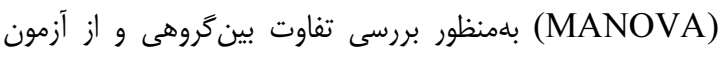

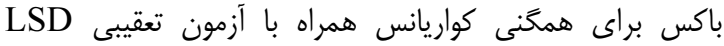

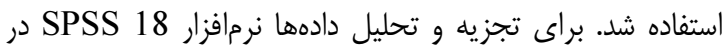
سطح معنى دارى كمتر از هـ/ مورد استفاده قرار كرفت.

يافتهها

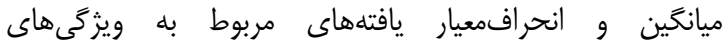

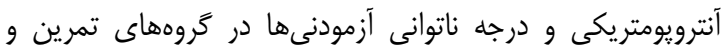
كنترل در جدول ا ارايه شده است.

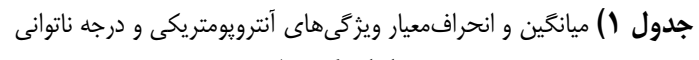
بلفتكيك گروهها

\begin{tabular}{|c|c|c|c|c|}
\hline ناتوانى & قد (سانتىمتر) & سن (سال) وزن (كيلوكَم) & \multicolumn{2}{|c|}{ تعداد (نفر) } \\
\hline & & & \multicolumn{2}{|c|}{ كروه تمرين } \\
\hline$r / V I \pm I / F)$ & $\mid V T / \cdot \cdot \pm \varepsilon / 4 \Lambda$ & $V E / r T \pm \mid r / . F \quad r V / V I \pm S / Q V$ & $\mathrm{v}$ & مرد \\
\hline$r / F \Delta \pm 1 / \cdot r$ & $10 S / / K \pm F / T r$ & 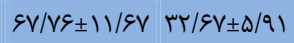 & ir & زن \\
\hline$r / \Delta \Delta \pm 1 / / \Delta$ & $\mid \varepsilon r / m r \pm q / V^{e}$ & $\varepsilon V / \cdot r \pm|r / \varphi \cdot r r / \Delta r \pm \varepsilon / \Delta|$ & 19 & كل \\
\hline & & & \multicolumn{2}{|c|}{ كروه كنترل } \\
\hline$r / A \gamma \pm 1 / r)$ & $\mid V E / V \Delta \pm F / F T$ & $V / / \cdot \Lambda \pm V \kappa / \cdot \leftarrow \leftarrow V V / \cdot \cdot \pm \Delta / \Delta$. & $\wedge$ & 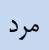 \\
\hline$r / q \cdot \pm \cdot / \Lambda \cdot$ & $\mid \Delta Q / \bar{g} \cdot \pm F / 19$ & $q \mu / \Delta \wedge \pm \mid l / r \cdot r q / \varepsilon \cdot \pm F / \eta)$ & 1. & زن \\
\hline$r / M_{ \pm} \cdot / q V$ & $\mid 94 / 11 \pm 1 . / 94$ & $g \varepsilon / Q r \pm 1 . / 1 r \quad r g / V \Lambda \pm r / q r$ & 11 & كل \\
\hline
\end{tabular}

همجنين ميانگين، انحرافمعيار و مقدار p براى مقياس شدت

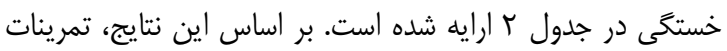
تركيبى باعث بهبود معنىدار در شدت خستخى بيماران MS شد. جدول ץ) تغييرات شدت خستخى (بر حسب امتياز) در كروهایى تمرين و كنترل

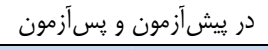

\begin{tabular}{|c|c|c|}
\hline تروه كنترل & تروه تمرين & متغيرها \\
\hline$r / r \pm \cdot / \Lambda r$ & $\varphi / 1 \cdot \pm 1 / 1$. & ميانكين نمرات در بيشىآزمون \\
\hline$r / r V \pm I / I r$ & $r / V \backslash \pm 1 / \cdot 0$ & ميانكين نمرات در پيسآزمون \\
\hline.$/ 49$ & $.1 \cdot .1$ & مقدار p درون تروهى \\
\hline ا & (كاهش) (ك/ه) & درصد تغيير \\
\hline \multicolumn{2}{|c|}{.$/ . .1$} & مقدار p بين كَروهى \\
\hline
\end{tabular}

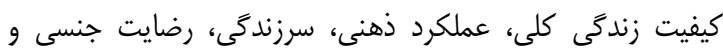

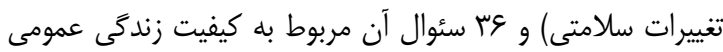

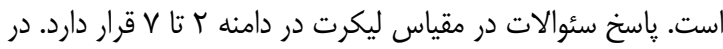

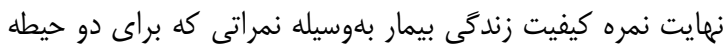

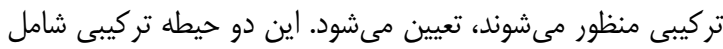

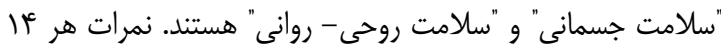

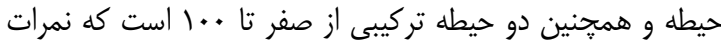

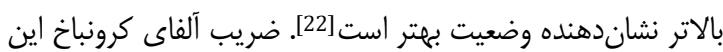
يرسشنامه در ايران وه/· گزارش شده است.

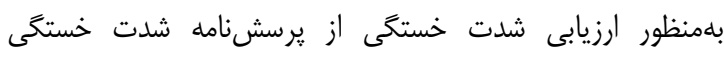

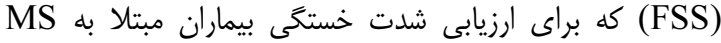

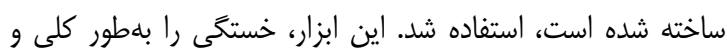

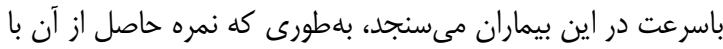

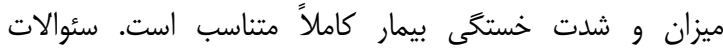

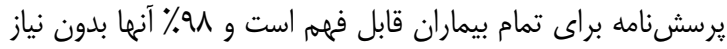

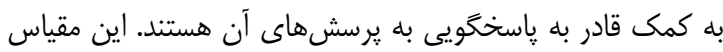

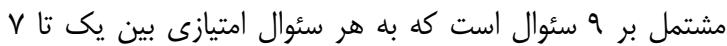

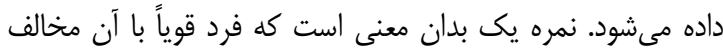

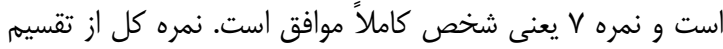

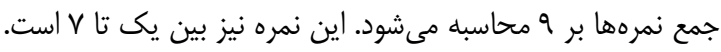

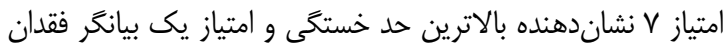

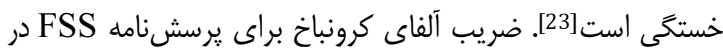

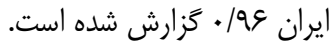

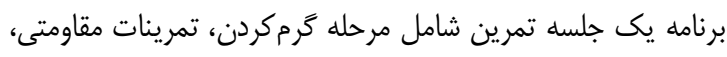

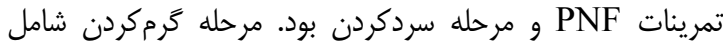
راهرفتن يا دوى نرم، حركات نرمشى و حر كات كششى بود. تمرينات

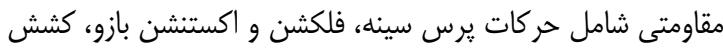

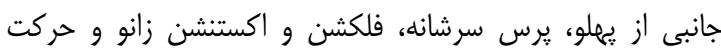

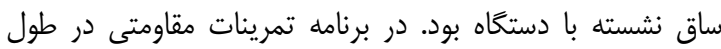

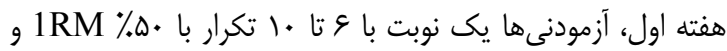

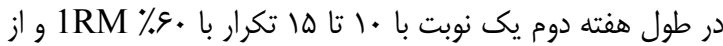

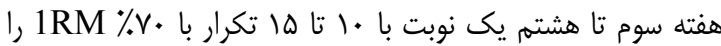

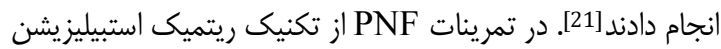

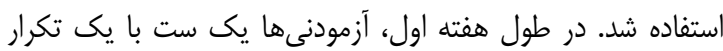

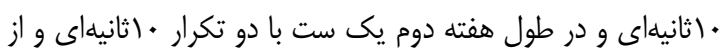
هفته سوم تا هشتم يك ست با سه تكرار •اثانيهاى را ان انجام

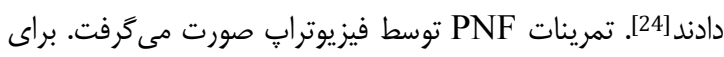

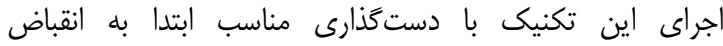

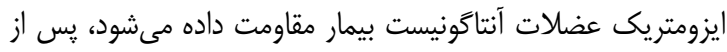

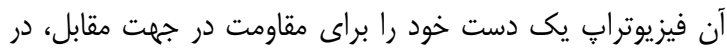

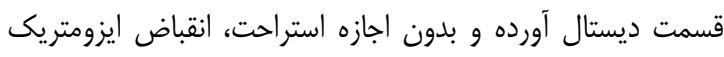

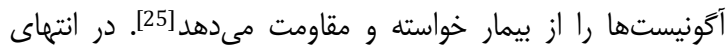




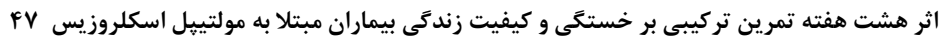

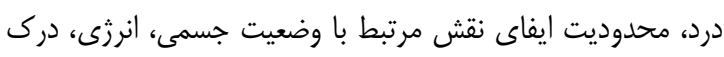

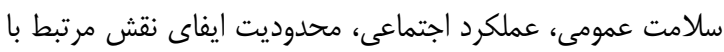
وضعيت روحى - روانى، سرزندگى، كيفيت زندگى كلى كلى و عملكرد

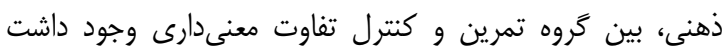

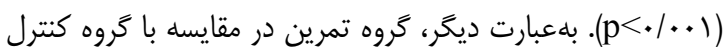

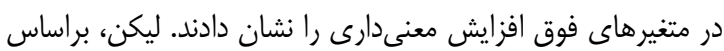
نتايج مشاهدهده، بين ميانخين نمرات تغييرات سلامتى، رضايت جنسى، تهديد سلامتى و عملكرد جنسى، بين گروه تمرين و كنترل

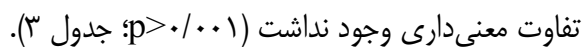

نتايج آزمون باكس نشان داد كه شرط همخنى ماتريسهاى

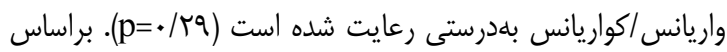

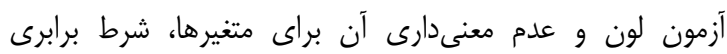

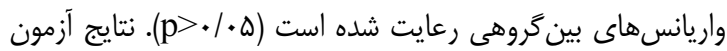

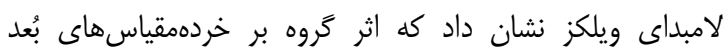

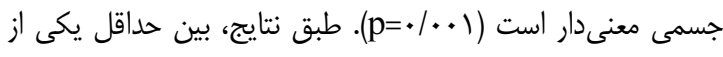
متغيرها در بين گروههاى مورد بررسى تفاوت معنىدارى وجود داشت. براساس نتايج بهدستآمده در جدول با، بين ميانخين نمرات

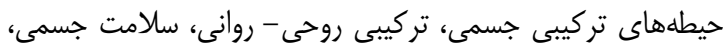

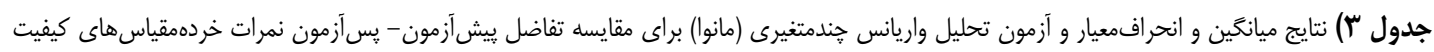

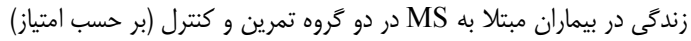

\begin{tabular}{|c|c|c|c|c|c|}
\hline $\mathbf{P}$ & F & MS & SS & كروه كنترل & كَروه تمرين \\
\hline $.1 . .1$ & $|r| \cdot|8|$ & $|V| r / M \Lambda$ & IVIr/M & $I V / \Lambda \Lambda \pm r / V V$ & 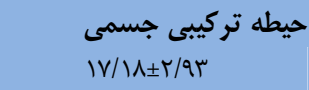 \\
\hline $.1 . .1$ & $V \times / 9 \Delta$ & IVVI/TS & $\mid W W / T \&$ & $r / r \Delta \pm \varepsilon / 11$ & حيطه تر كيبى روحى - روانى \\
\hline . & I/IAT & res/qqV & rFo/qqV & $\varepsilon / q \uparrow \pm 1 / / v q$ & تغييرات سلامتى . . . . \\
\hline . / equ &.$/ 011$ & IVQ/DTq & IVq/orq & $1 / r q \pm r \cdot / r \Delta$ & رضايت جنسى \\
\hline $.1 . .1$ & $99 / v \wedge$ & ITMT/VT & ITHT/VT & $1 / 94 \pm 4 / \wedge q$ & سلامت جسمى \\
\hline $.1 . .1$ & $\wedge r / \vee \wedge$ & rNDT/FT & rAST/AY & $1 / V \cdot \pm g / V V$ & $\mathrm{IV/Tq \pm \varepsilon /Vq}$ \\
\hline.$/ . r$ & $11 / 4$ & $r \Delta V T / \Delta T$ & rQVR/QT & جسمى & محدوديت ايفاى نقش مرتبط \\
\hline.$/ \cdot 1$ & ^®/৭น & $\mid M \mu F / \cdot r$ & $\mid M M F / \cdot F^{F}$ & $1 / \Delta \Delta \pm \Gamma / q 1$ & انرخى \\
\hline $.1 . .1$ & $|f V / A|$ & $r \cdot V N / T \Lambda$ & $r \cdot V N / r \Lambda$ & $1 / 11 \pm r / 99$ & 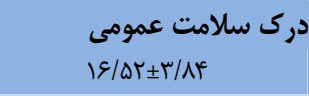 \\
\hline .1 .1 & $N \varepsilon / \Delta r$ & $0.9 \% / 1 \pi$ & $0.9 r / 1 r$ & $\cdot / F \vee \pm N / V q$ & 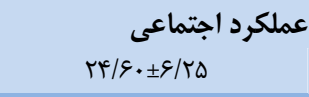 \\
\hline$\cdot 11 \cdot 1$ & $T / V T$ & $98 / 19$ & $98 / 19$ & $1 / \Gamma \Lambda \pm \xi / I \pi$ & تهديد سلامتى \\
\hline $.1 \cdot \mathrm{VA}$ & $r / \mu$. & rVq/M & rVq/MA & & عملكرد جنسى \\
\hline $.1 . .1$ & $\mathrm{IV} / \mathrm{VV}$ & eqRV/AD & FqTV/AD & 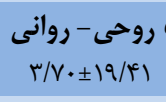 & محدوديت ايفاى نقش مرتبط \\
\hline $.1 \cdot 1$ & st/VV & $r r q 4 / 4$ & rrqf/r & & سرزند \\
\hline .1 .1 & $1 f q / \wedge f$ & DHTY $/ Q$ & DTrF/Q & $\cdot / r q \pm \Delta / \cdot r$ & كيفيت زندَّى كلى \\
\hline.$/ . r$ & $9 / 91$ & & GGT/KG & - $/ \Delta \Delta \pm \xi / \Lambda$ & عملكرد ذهنى \\
\hline
\end{tabular}




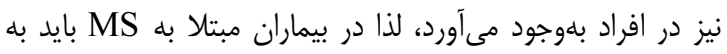
مدل تمرينى توجه خاصى شود تا انجام تمرينات ورزشى موجب دران افزايش خستخى در اين بيماران نشود [ناصن.

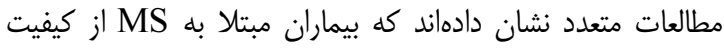

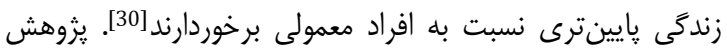

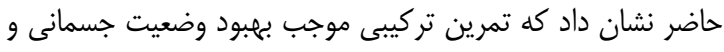

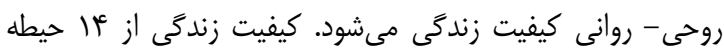

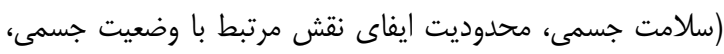

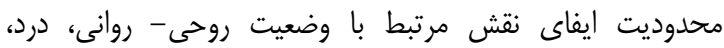

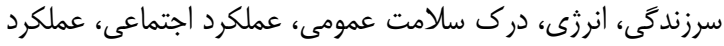

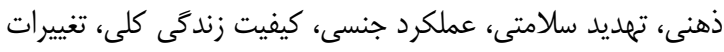

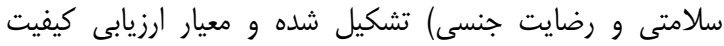

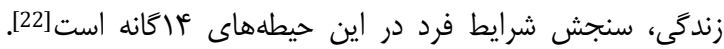

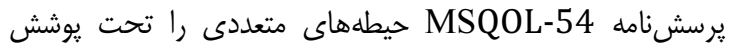

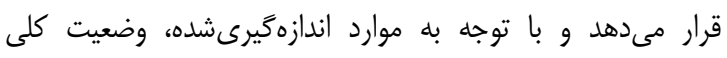

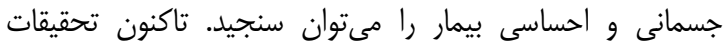

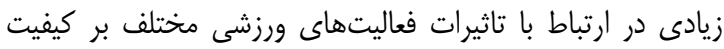

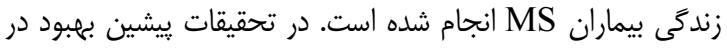

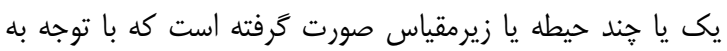

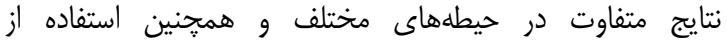

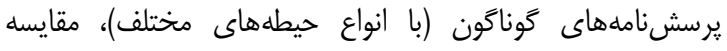

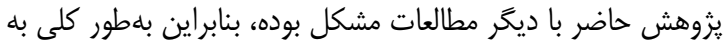

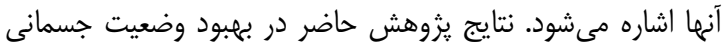

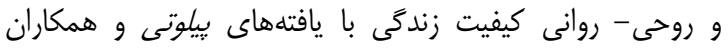

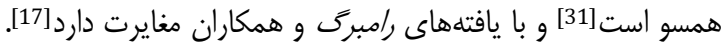
همجنين نتايج يزوهش حاضر، افزايش معنىدارى در بُعد سلامت

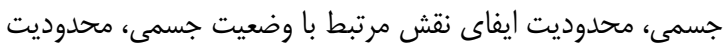

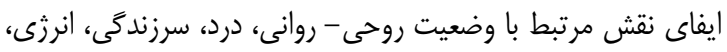

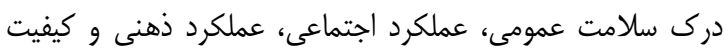

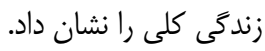

بجارنودوتير و همكاران به بررسى يك برنامه تمرينى با شدت

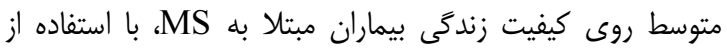

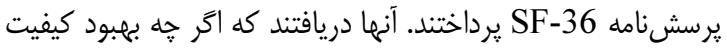

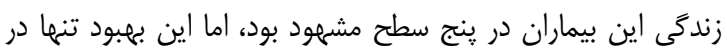

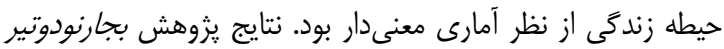

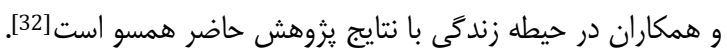

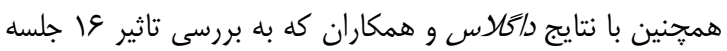

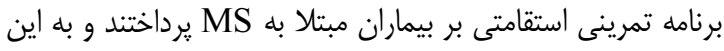

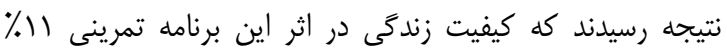

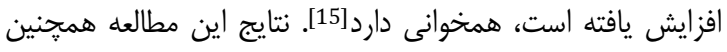

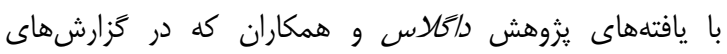
تحقيقى خود در مورد تاثير برنامه تمرينى قدرتى بر كيفيت زندگى إكى
يزوهش حاضر با هدف بررسى اثر يك برنامه تمرين تركيبى بر

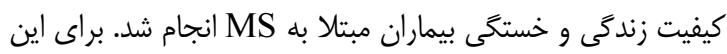

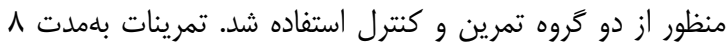

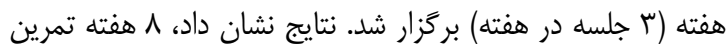

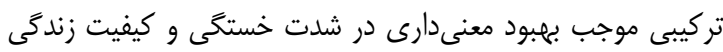

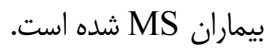

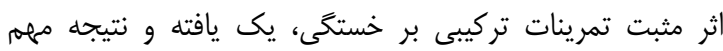

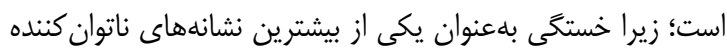

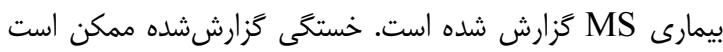

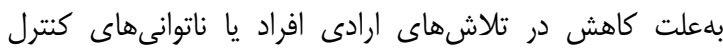

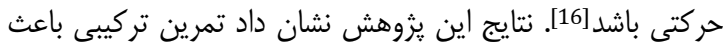

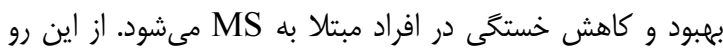

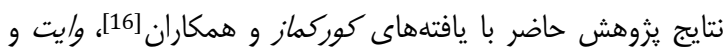

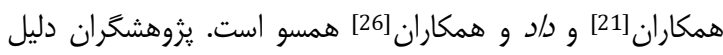

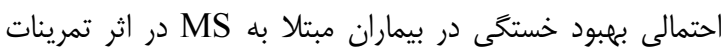

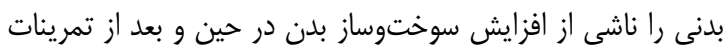

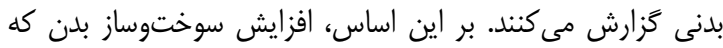

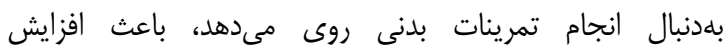
خونرسانى، اكسيثن و تغذيه بهتر اندامها و در نهايت باعث كانيات كاهش

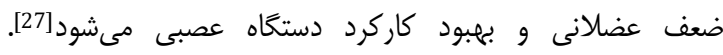

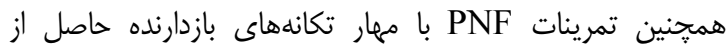

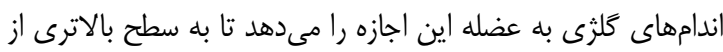

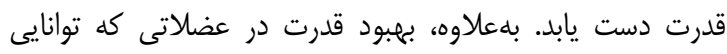

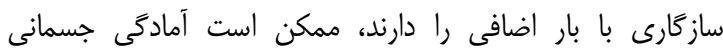
عمومى و توانايى عملكرد حركتى در مبتلايان به MS بارنا

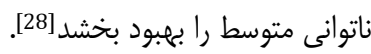

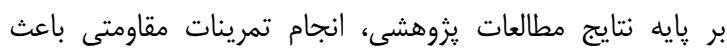

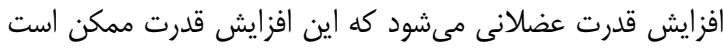

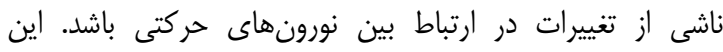

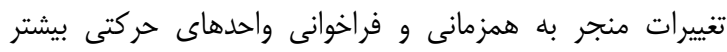

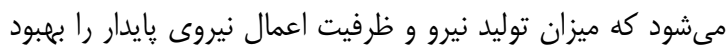

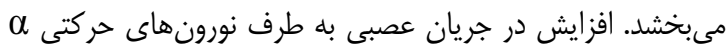

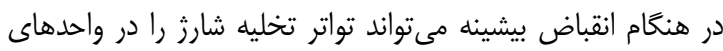

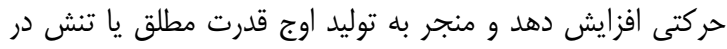

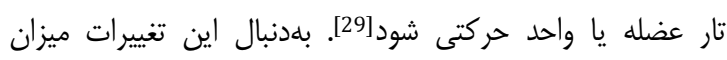

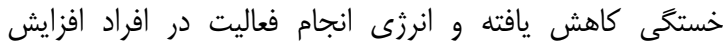

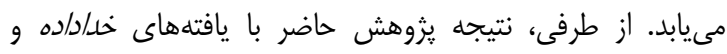

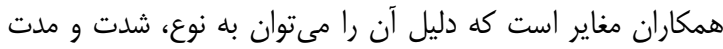

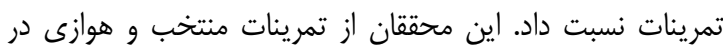

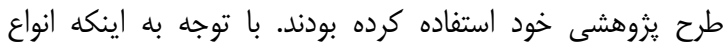

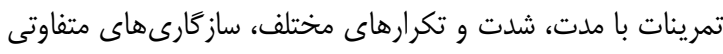

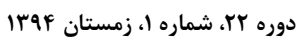

فصلنامه افق دانش 


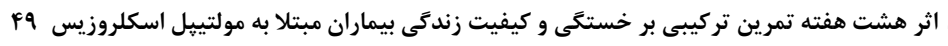

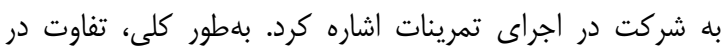
نتايج يِيشينهاى علمى و تحقيق حاضر را احتمالاً مى توان ناشى از

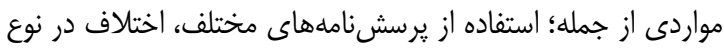
MS نوع برنامههاى تمرينى و حجم و شدت فعاليت ورزشى دانست.

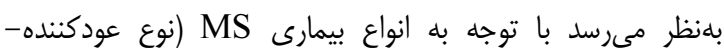

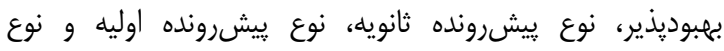

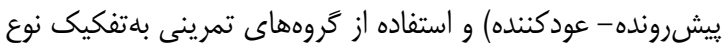
MS بيمارى مىتوان به نتايج دقيقتر و كاربردىترى براى بيماران

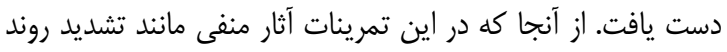
بيمارى MS در بين بيماران ديده نشده است، استفاده از اين تمرينات براى بيماران مبتلا به MS توصيه مي شئرد.

\section{نتيجه كيرى}

تمرين تركيبى مىتواند منجر به كاهش خستخى و بهبود در اكثر

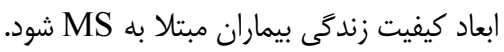

تشكر و قدردانى: نويسندكان لازم مىدانند از همكارى تمام

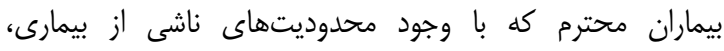
همكارى صميمانهاى با مجريان يثوهش داشتند، تشكر و قدردانى نمايند.

تاييديه اخلاقى: نويسندكان كليه كدهاى اخلاقى مربوط به تحقيقات روى نمونههاى انسانى را رعايت نمودند. تعارض منافع: موردى توسط نويسندگًان گَزارش نشده است.

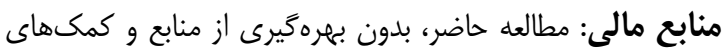

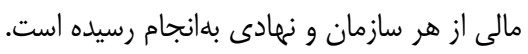

\section{منابع}

1- Holland NJ, Madonna M. Nursing grand rounds: Multiple sclerosis. J Neurosci Nurs. 2005;37(1):15-9.

2- Braunwald E, Fauci AS, Kasper DL, Hauser SL, Longo DL, Jameson JL. Harrison's principle of internal medicine. 15th edition. New York: McGraw-Hill Professional Publishing; 2001. pp. 2452-61.

3- Rasova K, Havrdova E, Brandejsky P, Zálisová M, Foubikova B, Martinkova P. Comparison of the influence of different rehabilitation programmes on clinical, spirometric and spiroergometric parameters in patients with multiple sclerosis. Mult Scler. 2006;12(2):227-34. 4- Huntley A, Ernst E. Complementary and alternative therapies for treating multiple sclerosis symptoms: A systematic review. Complement Ther Med. 2000;8(3):97-105.

5- Karpatkin HI. Multiple Sclerosis and exercise. Int J MS Care. 2005;7(2):36-41.

6- Yozbatiran N, Baskurt F, Baskurt Z, Ozakbas S, Idiman E. Motor assessment of upper extremity function and its relation with fatigue, cognitive function and quality of life in multiple sclerosis patients. J Neurol Sci. 2006;246(1-2):117-22.
بهبودهايى در حيطههاى مشكلات جسمانى، عاطفى و عملكرد

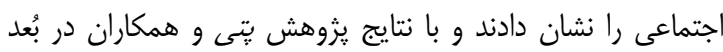

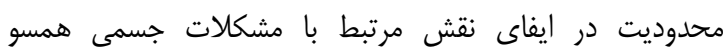

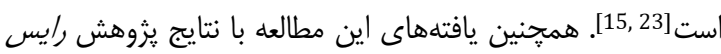
و همكاران همخوانى دارد، كه در ززارش تحقيقى خود نشان دادند

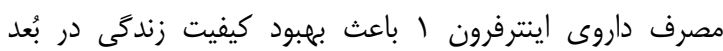

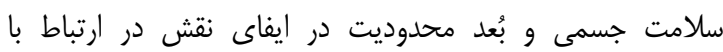

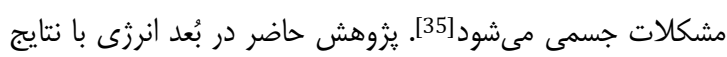

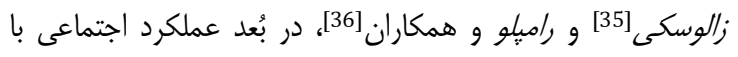

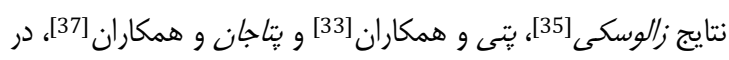

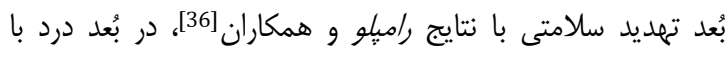

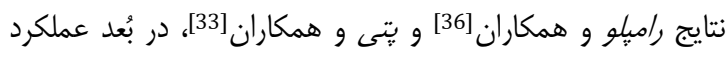

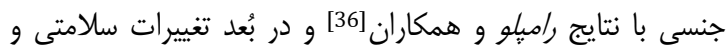

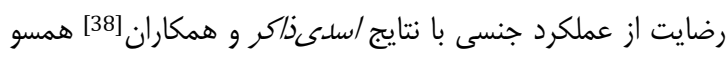

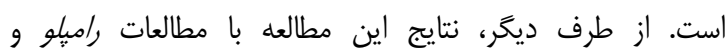
همكاران[36] و سيمونه و همكاران[39] كه نشان دادند تمرينات

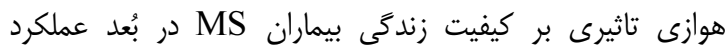

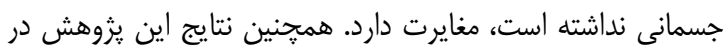

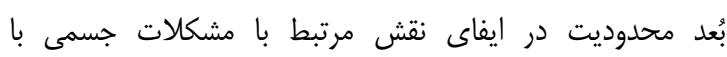

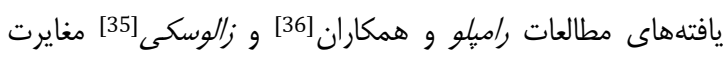

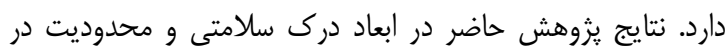
ايفاى نقش در ارتباط با مشكلات جسمى با نتايج راميلو و همكاران

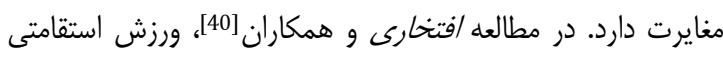
تنها باعث افزايش معنىدار در كيفيت زندگى كل، ابعاد سلامت دارت روانى و سلامت ذهنى شده است كه با نتايج مطالعه حاضر مغايرت

دارد.

اگر جه در تمامى تحقيقات انجامشده بهبود در كيفيت زندگى در

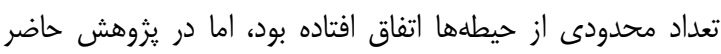

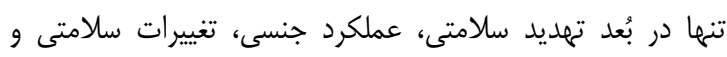
رضايت جنسى بهبود معنىدارى مشاهده نشده است. در بيماران

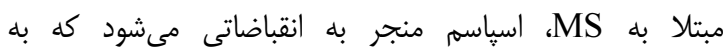

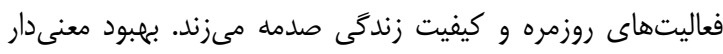

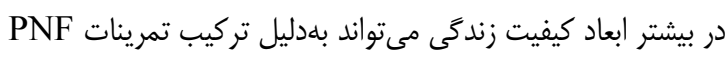

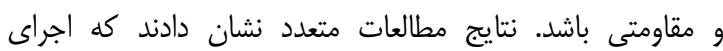
تمرينات PNF مىتواند باعث كاهش اسياسم در اين بيماران

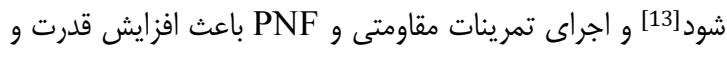
در نتيجه كاهش خستكى شود. همجنين اجراى تمرينات بدنى تمرئى مىتواند باعث افزايش رضايتمندى و اعتمادبانفس بيماران بهدليل شركت در فعاليتهاى بلدنى شود كه همه اين عوامل مىتواند تاعن تاثير مستقيمى بر كيفيت زندگى داشته باشد [15]. از محدودتهاى اين نزوهش مىتوان به نوع و مقدار داروهاى

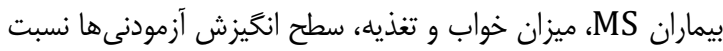


A pilot study. Neurorehabil Neural Repair. 2001;15(3):203-11.

25- Adler S, Beckers D, Buck M. PNF in practice. $3^{\text {rd }}$ edition. Berlin, Heidelberg: Springer-Verlag; 2008. pp. 67.

26- Dodd KJ, Taylor NF, Denisenko S, Prasad D. A qualitative analysis of a progressive resistance exercise programme for people with multiple sclerosis. Disabil Rehabil. 2006;28(18):1127-34.

27- De Groot MH, Phillips SJ, Eskes GA. Fatigue associated with stroke and other neurologic conditions: implications for stroke rehabilitation. Arch Phys Med Rehabil. 2003;84(11):1714-20.

28- Delecluse C, Roelants M, Verschueren S. Strength increase after whole-body vibration compared with resistance training. Med Sci Sports Exerc. 2003;35(6):1033-41.

29- Schulz KH, Gold SM, Witte J, Bartsch K, Lang UE, Hellweg R, et al. Impact of aerobic training on immuneendocrine parameters, neurotrophic factors, quality of life and coordinative function in multiple sclerosis. J Neurol Sci. 2004;225(1-2):11-8.

30- Tepavcevic DK, Kostic J, Basuroski ID, Stojsavljevic N, Pekmezovic T, Drulovic J. The impact of sexual dysfunction on the quality of life measured by MSQoL-54 in patients with multiple sclerosis. Mult Scler. 2008;14(8):1131-6.

31- Pilutti LA, Lelli DA, Paulseth JE, Crome M, Jiang S, Rathbone MP, et al. Effects of 12 weeks supported treadmill training on functional ability and quality of Life in progressive multiple sclerosis: A pilot study. Arch Phys Med Rehabil. 2011;92(1):31-6.

32- Hamidizadeh S, Masoodi R, Ahmadi FA, Mohammadi E. Evaluation of the effect of self-care program based on the orem framework on the physical quality of life in multiple sclerosis patients. J Shahid Sadoughi Univ Med Sci. 2009;17(2):153-62. [Persian]

33- Patti F, Ciancio MR, Reggio E, Lopes R, Palermo F, Cacopardo $\mathrm{M}$, et al. The impact of outpatient rehabilitation on quality of life in multiple sclerosis. J Neurol. 2002;249(8):1027-33.

34- Rice GP, Oger J, Duquette P, Francis GS, Bélanger M, Laplante $S$, et al. Treatment with interferon beta- $1 \mathrm{~b}$ improves quality of life in multiple sclerosis. Can J Neurological Sci. 1999;26(4):276-82.

35- Zalweski K. Exploring barriers to remaining physically active: a case report of a person with multiple sclerosis. J Neurol Phys Ther. 2007;31(1):40-5.

36- Rampello A, Franceschini M, Piepoli M, Antenucci R, Lenti G, Olivieri D, et al. Effect of aerobic training on walking capacity and maximal exercise tolerance in patients with multiple sclerosis: A randomized crossover controlled study. Phys Ther. 2007;87(5):545-55.

37- Petajan JH, Gappmaier E, White AT, Spencer MK, Mino L, Hicks RW. Impact of aerobic training on fitness and quality of life in multiple sclerosis. Ann Neurol. 1996;39(4):432-41.

38- Asadi Zaker M, Majdinasab N, Atapour M, Latifi SM, Babadi M. Effect of exercise on speed of walking, fatigue and quality of life in patients with multiple sclerosis. Sci J Ahwaz Univ Med Sci. 2010:9(2):189-98.

39- Simone IL, Ceccarelli A, Tortorella C, Bellacosa A, Pellegrini F, Plasmati I, et al. Influence of Interferon beta treatment on quality of life in multiple sclerosis patients. Health Qual Life Outcomes. 2006;4:96.

40- Eftekhari E, Nikbakht H, Rabiee K, Etemadifar M. The effect of endurance training on aerobic power and quality of life in women with multiple sclerosis. Olympic. 2008;16(1):37-46.
. ه آقابر اهيم عطار سياح و همكاران .

7- Janssens AC1, van Doorn PA, de Boer JB, Kalkers NF, van der Meche FG, Passchier J, et al. Anxiety and depression influence the relation between disability status and quality of life in multiple sclerosis. Mult Scler. 2003;9(4):397-403.

8- Raeesi $H$. The effect of education on self-care and the incidence of complications in patients with multiple sclerosis attending neurology clinics in the shiraz city in 2002 [Dissertation]. Faculty of Nursing \& Midwifery, University of Medical Sciences and Health Services of Iran; 2002.

9- Morgante L. Hope in multiple sclerosis: A nursing perspective. Int J MS Care. 2000;2(2):9-15.

10- Kishiyama S, Carlsen J, Lawrence J, Small E, Zajdel D, Oken BS. Yoga as an experimental intervention for cognition in MS. Int J Yoga Ther. 2002;(12):57-62.

11- Bonomi AE, Patrick DL, Bushnell DM, Martin M. Validation of the United States version of the World Health Organization Quality of Life (WHOQOL) instrument. J Clin Epidemiol. 2000;53(1):1-12.

12- Zifko UA. Therapy of day time fatigue in patients with multiple sclerosis. Wien Med Wochenschr. 2003;153(34):65-72. [German]

13- Henze T, Rieckmann P, Toyka KV. Symptomatic treatment of multiple sclerosis. Multiple sclerosis therapy consensus group (MSTCG) of the German multiple sclerosis society. Eur Neurol. 2006;56(2):78-105. 14- Miller A, Dishon S. Health-related quality of life in multiple sclerosis: The impact of disability, gender and employment status. Qual Life Res. 2006;15(2):259-71. 15- Daglas U, Stenager E, Ingemann-Hansen T. Multiple sclerosis physical exercise: Recommendations for the application of resistance-, endurance- and combined training. Mult Scler. 2008;14(1):35-53.

16- Korkmaz NC, Kirdi N, Temucin CM, Armutlu K, Yakut Y, Karabudak R. Improvement of muscle strength and fatigue with high voltage pulsed galvanic stimulation in multiple sclerosis patients- a non-randomized controlled trial. J Pak Med Assoc. 2011;61(8):736-43.

17- Romberg A, Virtanen A, Ruutiainen J. Long-term exercise improves functional impairment but not quality of life in multiple sclerosis. J Neurol. 2005;252(7):839-45. 18- Khodadadeh S, Kordi MR, Khosravi N, Sangolji B. Effect of 8-week selected training on distance walking, strength and fatigue in multiple sclerosis patients. $7^{\text {th }}$ International Congress on Physical Education and Sport Sciences; 2009.

19- Sliwa JA. Neuromuscular rehabilitation and electrodiagnosis. 1. Central neurologic disorders. Arch Phys Med Rehabil 2000;81(3 Suppl 1):S3-12.

20- Capodaglio P, Facioli M, Burroni E, Giordano A, Ferri A, Scaglioni G. Effectiveness of a home-based strengthening program for elderly males in Italy. A preliminary study. Aging Clin Exp Res. 2002;14(1):28-34. 21- White LJ, McCoy SC, Castellano V, Gutierrez G, Stevens JE, Walter GA, et al. Resistance training improves strength and functional capacity in persons with multiple sclerosis. Mult Scler. 2004;10(6):668-74.

22- Ghaem H, Borhani Haghighi A, Jafari P, Nikseresht AR. Validity and reliability of the Persian version of the multiple sclerosis quality of life questionnaire. Neurol India. 2007;55(4):369-75.

23- Basampoor ShS, Nikbakht Nasrabadi AR, Faghihzadeh S, Monjazebi F. Assess the using and efficacy of fatigue reducing strategies in patients referred to multiple sclerosis association of Iran. J Hayat. 2005;11(4-3):29-37. [Persian]

24- Armutlu K, Karabudak R, Nurlu G. Physiotherapy approaches in the treatment of ataxic multiple sclerosis:

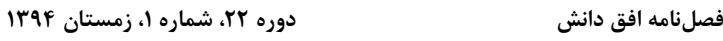

